\title{
Approach to Fever in the Returning Traveler
}

\author{
Guy E. Thwaites, F.R.C.P., and Nicholas P.J. Day, F.Med.Sci., F.R.C.P.
}

From the Centre for Tropical Medicine and Global Health, Nuffield Department of Medicine, University of Oxford, Oxford, United Kingdom (G.E.T., N.P.J.D.); Oxford University Clinical Research Unit, Ho Chi Minh City, Vietnam (G.E.T.); and the Mahidol-Oxford Tropical Medicine Research Unit, Faculty of Tropical Medicine, Mahidol University, Bangkok, Thailand (N.P.J.D.). Address reprint requests to Prof. Thwaites at Oxford University Clinical Research Unit, 764 Vo Van Kiet, Quan 5, Ho Chi Minh City, Vietnam, or at gthwaites@oucru.org.

N EnglJ Med 2017;376:548-60. DOI: 10.1056/NEJMral508435

Copyright @ 2017 Massachusetts Medical Society.
1 EVER IN THE RETURNING TRAVELER IS A COMMON CLINICAL SCENARIO that often leads to hospitalization and may be the only symptom of a serious or life-threatening illness. ${ }^{1}$ Three percent of 784 Americans who traveled abroad for short periods reported an episode of febrile illness, ${ }^{2}$ and fever was the chief symptom in $28 \%$ of 24,920 ill travelers who presented to travel clinics on their return home. ${ }^{3}$ The absolute number of travelers is large and rising, with the International Tourism Organization reporting 1.2 billion trips in 2015, an increase of $4.4 \%$ from the previous year. ${ }^{4}$ The challenge presented by returning travelers with febrile illnesses is changing for two reasons. First, increasing numbers of travelers are older than 60 years of age or are seeking health care elsewhere ("medical tourists"), and these travelers are more likely than others to have clinically significant coexisting conditions and consequently increased morbidity from infections. Second, the likelihood of multidrug resistance in the infecting organisms is increasing. ${ }^{5-7}$ The recent Ebola epidemic in West Africa, the emergence of the Middle East respiratory syndrome coronavirus (MERS-CoV), and the reemergence of Zika and chikungunya viruses have highlighted the importance of being alert to the possibility that an emerging pathogen is causing a febrile episode.

Fever in the returning traveler is an evolving clinical challenge, with respect to both the infections responsible for the fever and the sources and quality of information available to assist the physician. We review available sources of global information on outbreaks and the epidemiologic features of infectious diseases and offer a practical approach to emerging or transmissible infectious diseases that may pose a life-threatening risk to patients, as well as clinicians and laboratory workers.

SURVEILLANCE AND SOURCES OF INFORMATION

A detailed travel history is central to the assessment of the febrile returning traveler. Its value, however, depends on accurate, up-to-date, and rapidly accessible information about the possible infections acquired in the places visited. There are a bewildering number of sources of information concerning the geographic risk of various infectious agents; an overview is provided in Table S1 in the Supplementary Appendix, available with the full text of this article at NEJM.org. The Centers for Disease Control and Prevention (CDC) website summarizes the global epidemiologic features of the key travel-related infections and provides essential information on the geographic risks of malaria.

Disease surveillance among travelers provides an important source of information that can inform advice before travel and clinical management after travel. ${ }^{8}$ Furthermore, surveillance among travelers can identify sentinels for outbreaks of new diseases and can be used to track the global movement of infectious diseases. ${ }^{9}$ Collaborative networks of travel clinics have been developed as a powerful surveillance tool; GeoSentinel is the preeminent example. Established in 1995 by the Inter- 
national Society of Travel Medicine and the CDC, GeoSentinel consists of 63 travel clinics on six continents that contribute Web-based, real-time data on ill travelers to a central database. ${ }^{10}$ Ongoing trends are tracked on a month-to-month basis for 60 key diagnoses, with additional syndromic surveillance. Quarterly reports are generated centrally for participating sites, and published scientific articles report longer-term trends. ${ }^{11}$ Linked surveillance networks exist in Canada (CanTravNet) ${ }^{12}$ and Europe (EuroTravNet). ${ }^{13}$ All these networks share the same limitations of sampling and selection bias, since they typically include information on travelers seen at an institution with a linked participating clinic, and many of the clinics are in specialized academic centers. Incidence rates and absolute risks cannot be calculated without estimates of denominators and missing cases.

Novel surveillance methods provide a valuable additional source of information. Automated newsscanning software can enable early detection of outbreaks. ${ }^{14}$ For example, HealthMap successfully tracked the 2009 H1N1 global influenza outbreak using multilingual data from news wires, media websites, RSS (Really Simple Syndication) feeds, mailing lists such as ProMED, and authoritative sources such as the World Health Organization (WHO), the CDC, and the European Centre for Disease Prevention and Control. ${ }^{15}$

Advanced clinical decision-making tools are also available to assist physicians. Swiss guidelines for primary care physicians on the management of febrile illness in travelers are available through a Web-based diagnostic algorithm (www.fevertravel .ch).$^{16} \mathrm{~A}$ recent evaluation involving 539 physicianpatient pairs showed approximately $40 \%$ adherence to the provided guidance per case and good clinical outcomes. ${ }^{17}$ KABISA, developed by the Institute of Tropical Medicine of Antwerp, Belgium, is another clinical decision-making support system that is available free of charge for the diagnosis of febrile illnesses after tropical travel. In a study involving 205 patients, KABISA performed as well as expert travel physicians in diagnosing febrile illnesses, often providing unconsidered diagnoses. ${ }^{18}$ The Global Infectious Diseases and Epidemiology Network (GIDEON) offers a commercial computer software program that uses a Bayesian matrix to generate a differential diagnosis on the basis of a patient's travel history, the clinical and laboratory findings, and the incubation periods for possible infections. ${ }^{19}$ Inde- pendent evaluations of the program's performance suggest that it provides a reasonably accurate differential diagnosis that may alert inexperienced physicians, in particular, to unconsidered infections. ${ }^{20-23}$

\section{CLINICAL APPROACH}

The possible causes of fever in the returning traveler are legion, and a specific diagnosis is often difficult to establish because diagnostic tests for many diseases either perform poorly or are not available locally. Even at referral centers with diagnostic expertise, approximately $25 \%$ of patients never receive a diagnosis (though they generally do well clinically). ${ }^{3}$ For this reason, a risk-based approach is advisable, with initial priority given to identifying and treating life-threatening causes of fever and those posing a high risk of transmission to health care workers, laboratory staff, and the wider population (Fig. 1).

\section{RECOGNIZING LIFE-THREATENING CAUSES OF FEVER}

Among 82,825 cases of illness in travelers that were reported to GeoSentinel between 1996 and 2011, a total of 3655 cases (4\%) involved acute and potentially life-threatening tropical diseases, and fever was a symptom in $91 \%$ of these cases ${ }^{11}$ (Table 1). Falciparum malaria accounted for $77 \%$ of the 3655 cases, and enteric fever for $18 \%$. Thirteen patients $(0.4 \%)$ died: 10 with falciparum malaria, 2 with melioidosis, and 1 with severe dengue. Falciparum malaria was acquired mainly in West Africa, and enteric fever was contracted largely on the Indian subcontinent. Travelers in this study had predominantly visited developing countries and were seen at travel clinics, characteristics that skewed the results. For the examining clinician, more widely distributed "cosmopolitan" infections that cause severe febrile illness should not be overlooked, including respiratory and urinary tract infections, meningococcal disease, and tuberculosis. Even among travelers returning from the tropics, "nontropical" causes of fever are common, with a rate of $34 \%$ reported in one European case series. ${ }^{25}$

Morbidity, including rates of hospitalization with febrile illness, and mortality are greater among elderly travelers than among those who are younger. ${ }^{5,25}$ In most case series involving fever in returning travelers, deaths have been uncommon, with overall mortality ranging from 0.2 to 


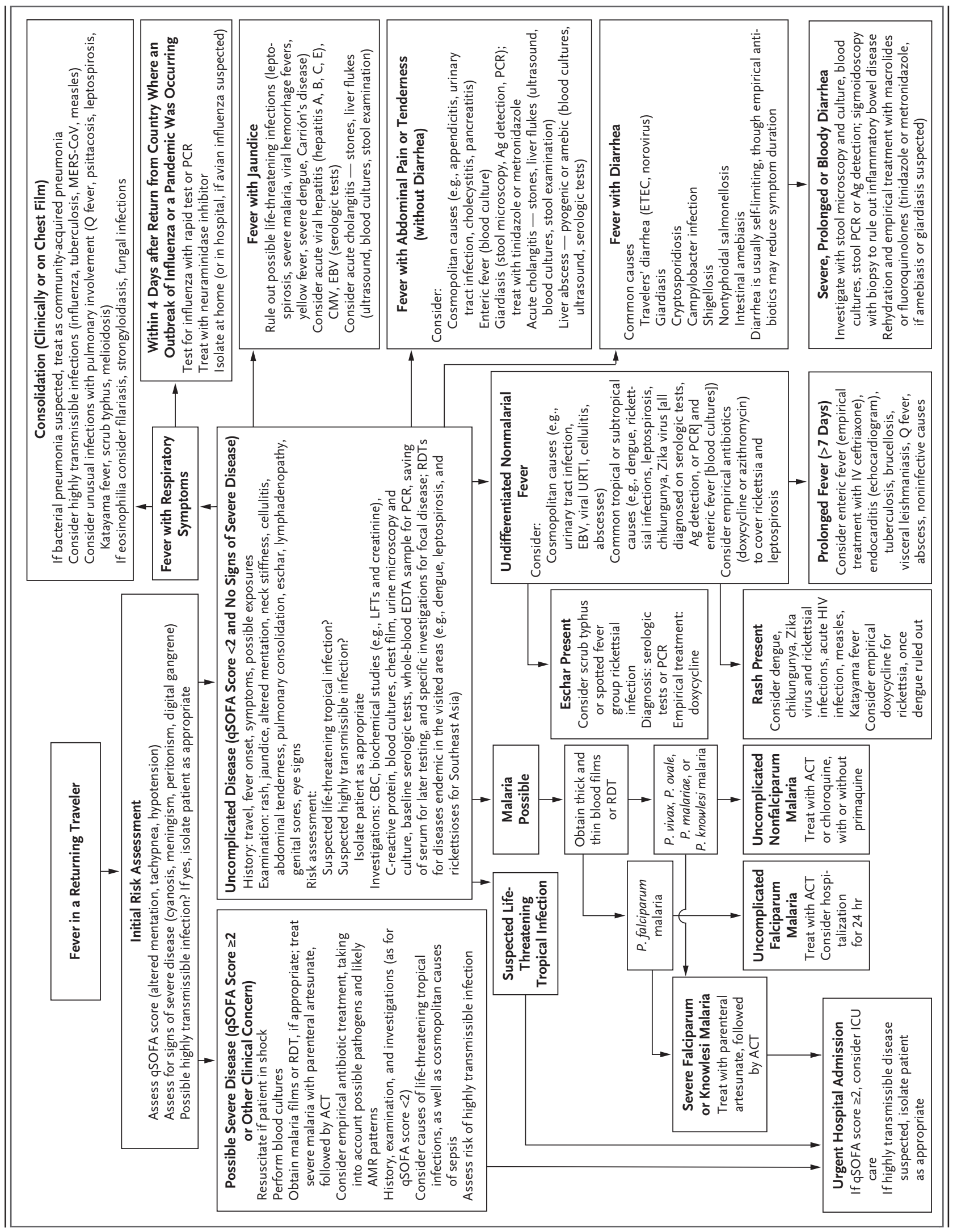


Figure 1 (facing page). A Risk-based Approach to Fever in the Returning Traveler.

A positive result of a rapid diagnostic test (RDT) for malaria should always be confirmed by assessing thick and thin blood films. After glucose-6-phosphate dehydrogenase (G6PD) testing to rule out G6PD deficiency, give primaquine for a radical cure if Plasmodium vivax or $P$. ovale infection is confirmed. The Quick Sepsisrelated Organ Failure Assessment (qSOFA) score ranges from 0 to 3 , with 1 point for each of the following findings: low blood pressure (systolic pressure $\leq 100 \mathrm{~mm} \mathrm{Hg}$ ), high respiratory rate ( $\geq 22$ breaths per minute), and altered mentation (a score of $<15$ on the Glasgow Coma Scale, which ranges from 3 to 15, with lower scores indicating reduced levels of conscious ness). ACT denotes artemisinin-based combination therapy, Ag antigen, AMR antimicrobial drug resistance, CBC complete blood count, CMV cytomegalovirus, EBV Epstein-Barr virus, ETEC enterotoxigenic Escherichia coli, ICU intensive care unit, IV intravenous, LFT liver-function tests, MERS-CoV Middle East respiratory syndrome coronavirus, PCR polymerase chain reaction, and URTI upper respiratory tract infection. Adapted from Gherardin and Sisson. ${ }^{24}$

$0.5 \% \cdot{ }^{3,25,26}$ Falciparum malaria is the most common serious infection seen in returning travelers and remains the main cause of death. In areas where falciparum malaria is not endemic, a delay in diagnosis is common and may have fatal consequences. During the recent Ebola epidemic in West Africa, falciparum malaria was the most common diagnosis in patients returning from the affected area. ${ }^{12}$ Other reported causes of death include melioidosis, severe dengue, scrub typhus, enteric fever, encephalitis, and nontropical infections, including influenza, bacterial pneumonia, and septicemia. ${ }^{5}$

Initial assessment of the patient should be the same as that for any ill patient suspected of having an infection. A full history should be obtained, with a view to determining exposure risks, risks associated with particular geographic areas, and likely incubation periods (Table 2). A thorough physical examination should be performed, with particular attention to signs of severity, a rash, and an infectious focus such as a chest infection or jaundice indicating hepatitis. The Quick Sepsisrelated Organ Failure Assessment (qSOFA), recommended recently on the basis of the Third International Consensus Definitions for Sepsis and Septic Shock (Sepsis 3), can be used as part of a rapid clinical assessment to identify patients at risk for severe sepsis and in need of high levels of care and urgent empirical treatment with antibiotics (i.e., patients meeting two or more of the following criteria: altered mentation, respiratory rate $\geq 22$ breaths per minute, and systolic blood pressure $\leq 100 \mathrm{~mm} \mathrm{Hg}){ }^{27}$ An eschar should be specifically sought; for travelers returning from southern Africa, one or more eschars may point to the relatively benign African tick typhus (caused by Rickettsia africae), but for those returning from South or Southeast Asia, an eschar suggests scrub typhus (Orientia tsutsugamushi infection), a potentially fatal disease. ${ }^{28}$ The eschar of scrub typhus is often missed, because the mite vector frequently bites in moist areas of the body that are usually covered, such as the genitalia, the perineum, and the area beneath the breasts. ${ }^{29}$

As a priority, malaria should be actively ruled out in all travelers returning from regions where it is endemic, regardless of whether they have been receiving chemoprophylaxis (Fig. 1). Thick and thin blood smears should be tested, with testing repeated twice if the results are negative. Malaria can be diagnosed within minutes when rapid diagnostic tests are performed either at the bedside or in the laboratory. These tests are particularly useful in areas where local expertise in microscopic examination for malaria is in short supply, though the result should be confirmed by assessment of a blood smear. Patients with malaria who are prostrate or comatose; those who have shock, acidosis, severe anemia, hypoglycemia, evidence of vital organ dysfunction, or a high parasitemia level $(>10 \%)$; and those who are unable to take oral medications reliably should be treated for severe malaria. In adults, children, and pregnant women, severe malaria should be treated promptly with parenteral artesunate, which has been shown to reduce mortality substantially as compared with quinine (and by extension quinidine)..$^{30,31}$ This is followed by a course of oral artemisinin-based combination therapy (ACT) when oral medication can be taken reliably. The Food and Drug Administration has not approved the use of artesunate in the United States, but it is available through the CDC Malaria Hotline. ${ }^{32}$

Empirical treatment of patients with suspected life-threatening infections should be driven by the clinical picture and likely exposure. If severe sepsis is suspected, local and national guidelines should be followed, with modifications for any differences in the prevalence of antimicrobial resistance in the geographic location visited. For example, Entero- 


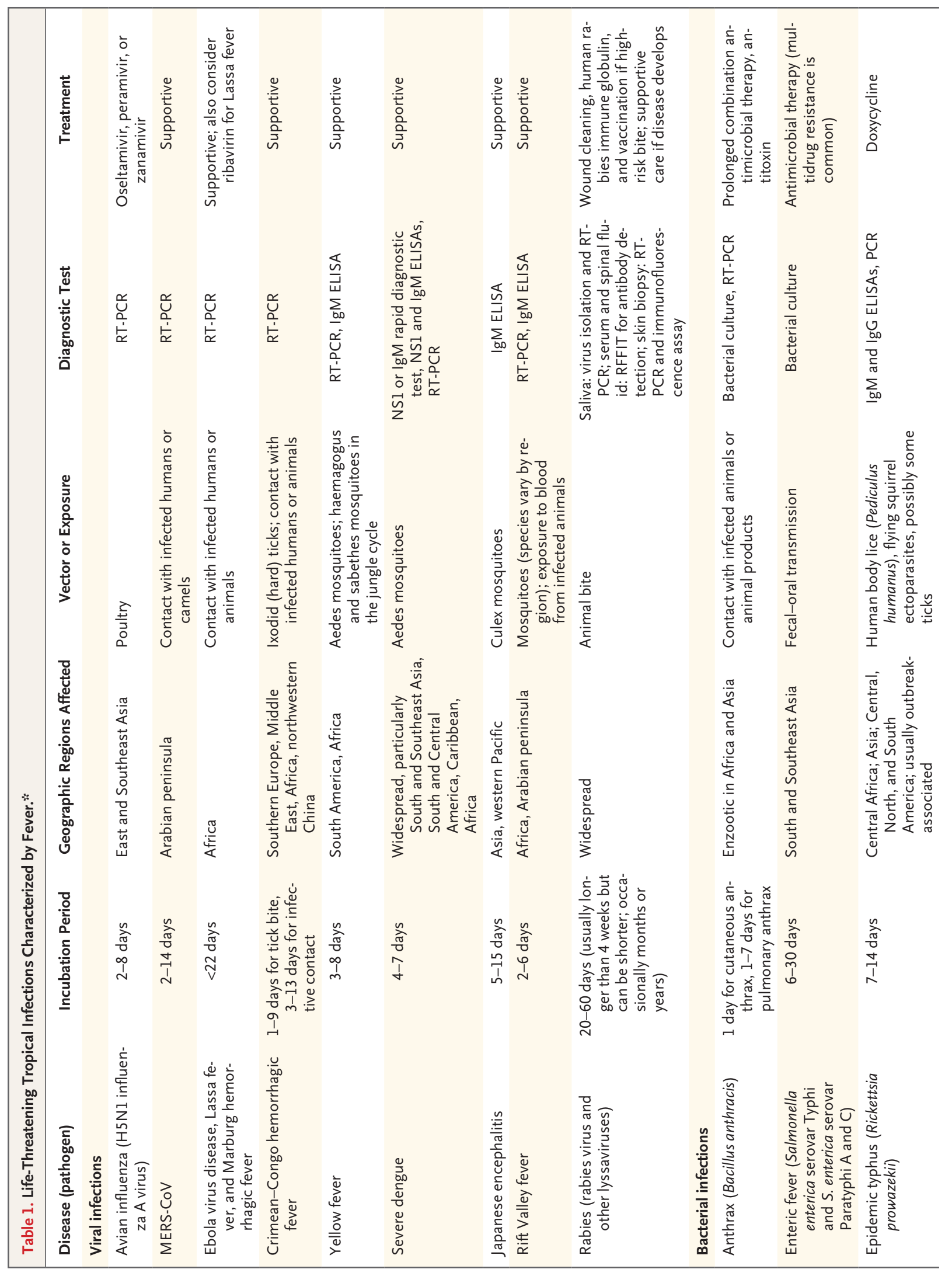




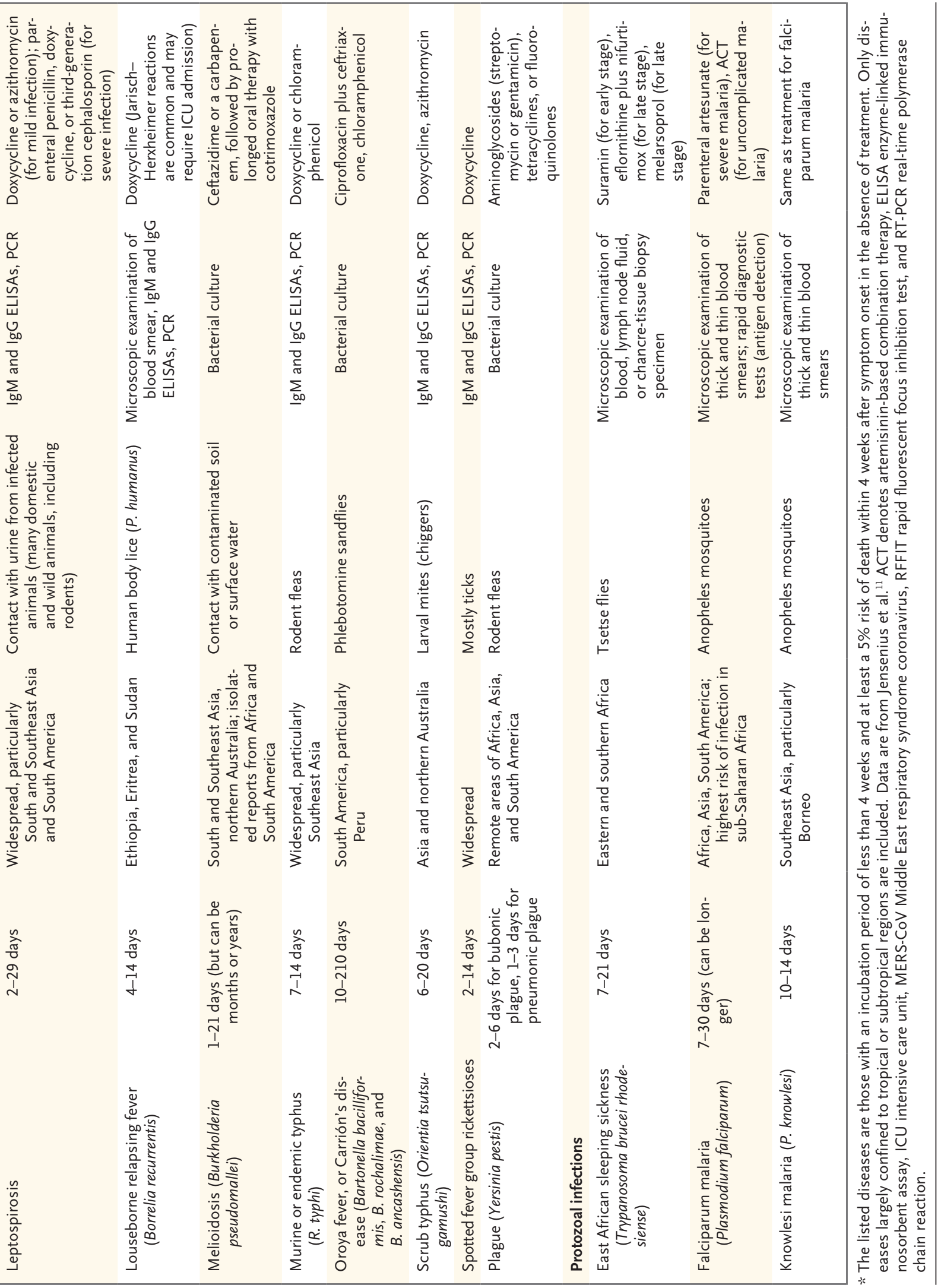




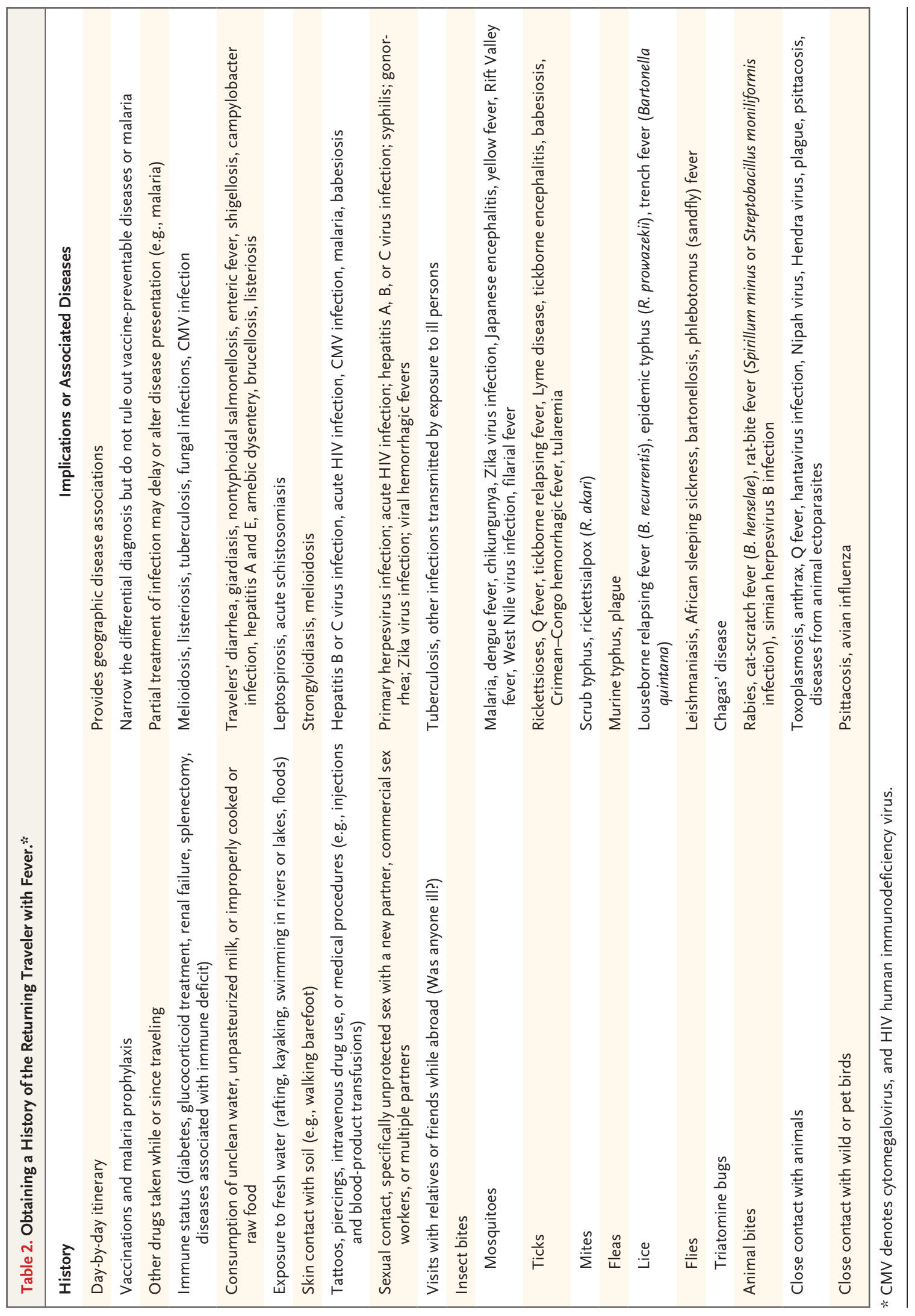


bacteriaceae that produce extended-spectrum betalactamase (ESBL) are highly prevalent in many developing countries, and travelers to such countries are at risk for colonization with these resistant bacteria, particularly if the travelers were hospitalized or received antibiotic treatment for traveler's diarrhea. ${ }^{33}$ If local guidelines for the treatment of community-acquired sepsis do not include a carbapenem, this should be given if the patient has traveled to an area where ESBL is highly prevalent, such as South or Southeast Asia. Similarly, melioidosis (caused by the environmental bacterium Burkholderia pseudomallei) is a common cause of sepsis in parts of Southeast Asia, and suspected infection should be treated with a carbapenem or ceftazidime, since the bacteria are intrinsically resistant to most beta-lactam antibiotics and to aminoglycosides. ${ }^{34}$ If severe scrub typhus (or another rickettsial infection) is suspected, doxycycline should be added to the empirical regimen, since 0 . tsutsugamushi and rickettsia species are intrinsically resistant to beta-lactams..$^{35}$

\section{RECOGNIZING HIGHLY TRANSMISSIBLE CAUSES OF FEVER}

A risk assessment for highly transmissible pathogens is a critical first step in the approach to the febrile traveler (Table 3 and Fig. 1). Categorizing risk allows for appropriate precautions to be taken that are aimed at protecting health care and laboratory workers, other patients, visitors, and the wider community from acquiring life-threatening infections. Viral hemorrhagic fevers such as Ebola, Crimean-Congo hemorrhagic fever, Marburg hemorrhagic fever, and Lassa fever must be considered because of the high associated mortality and potential for human-to-human transmission with nosocomial outbreaks. ${ }^{36}$ But infections transmitted by droplets or aerosols, such as influenza, MERS-CoV infection, measles, and tuberculosis, should also be considered, as should colonization or infection with antibiotic-resistant organisms such as methicillin-resistant Staphylococcus aureus or carbapenem-resistant Enterobacteriaceae.

Risk-assessment algorithms published by Public Health England and the CDC note that viral hemorrhagic fever should be suspected in patients who have fever (temperature, $\geq 37.5^{\circ} \mathrm{C}$ ) or a history of fever in the previous 24 hours and who have traveled within 21 days to an area where viral hemorrhagic fever is endemic or epidemic. ${ }^{37,38}$ Sources of information on affected countries and regions are listed in Table S1 in the Supplementary Appendix. The level of risk is defined according to the exposure history, but direct contact with body fluids from people or animals known or suspected to have a viral hemorrhagic fever constitutes high risk. Sexual contact with an infected person and funeral attendance may also increase exposure and the risk of infection from Ebola, Lassa, and Marburg viruses. ${ }^{39-41}$

Malaria remains the most likely diagnosis in patients with suspected viral hemorrhagic fever, ${ }^{12}$ and blood films and other essential tests (blood cultures, a complete blood count, and tests of kidney and liver function) should not be delayed pending the results of tests for viral hemorrhagic fever, which are usually performed by specialist reference laboratories. Any laboratory should be informed, however, of specimens submitted from patients with possible viral hemorrhagic fever. Public Health England recommends that these specimens be analyzed with the use of biosafety level 2 laboratory procedures and autoanalyzers; standard precautions should also be taken.

Outbreaks of severe respiratory viral infections during the past decade, such as those involving influenza viruses (H5N1, H7N9, and H1N1) and MERS-CoV, as well as the severe acute respiratory syndrome,$^{42}$ have exemplified the role of travelers as sentinels, carriers, and transmitters of these infections and the need for continued vigilance for novel respiratory pathogens. Knowledge of current outbreaks is essential for identifying cases early and reducing transmission risks. More familiar and potentially highly transmissible infections, such as measles, chicken pox, and tuberculosis, also require prompt consideration, especially in hospitals because of the proximity of potentially vulnerable patients.

Colonization or infection with antibiotic-resistant bacteria may complicate treatment and spread to others. Travel to tropical regions is associated with the transient acquisition of antibiotic-resistant commensal bacteria in the gastrointestinal tract, lasting around 3 months, ${ }^{43}$ and the globalization of health care, including the development of medical tourism, has created new risks for patients and hospitals. Planned or emergency inhospital care in one country, followed by the transfer of care to another country, has the potential to fuel the rapid global dissemination of highly antibiotic-resistant organisms. ${ }^{44}$

Patients should be isolated in a single room 


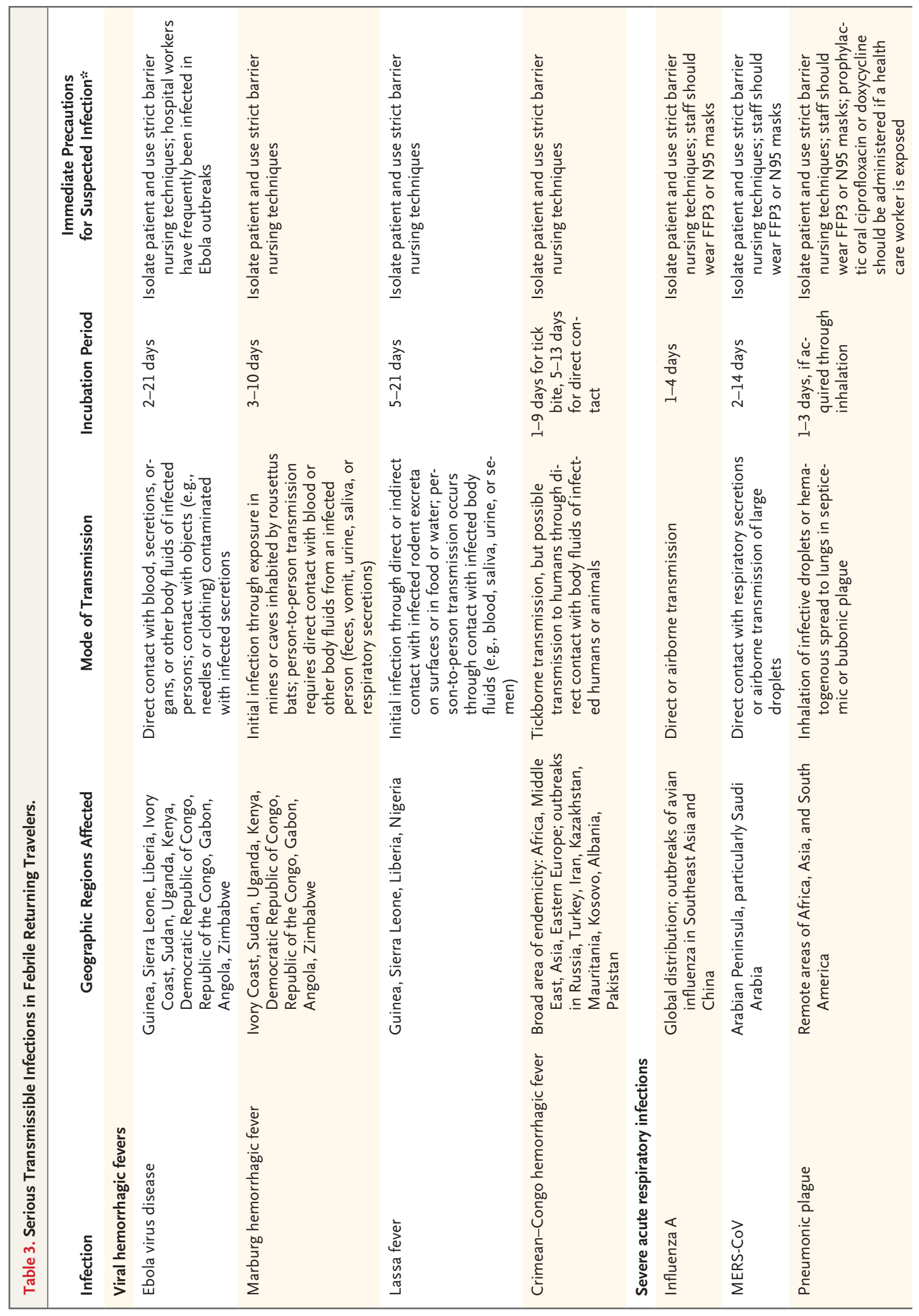




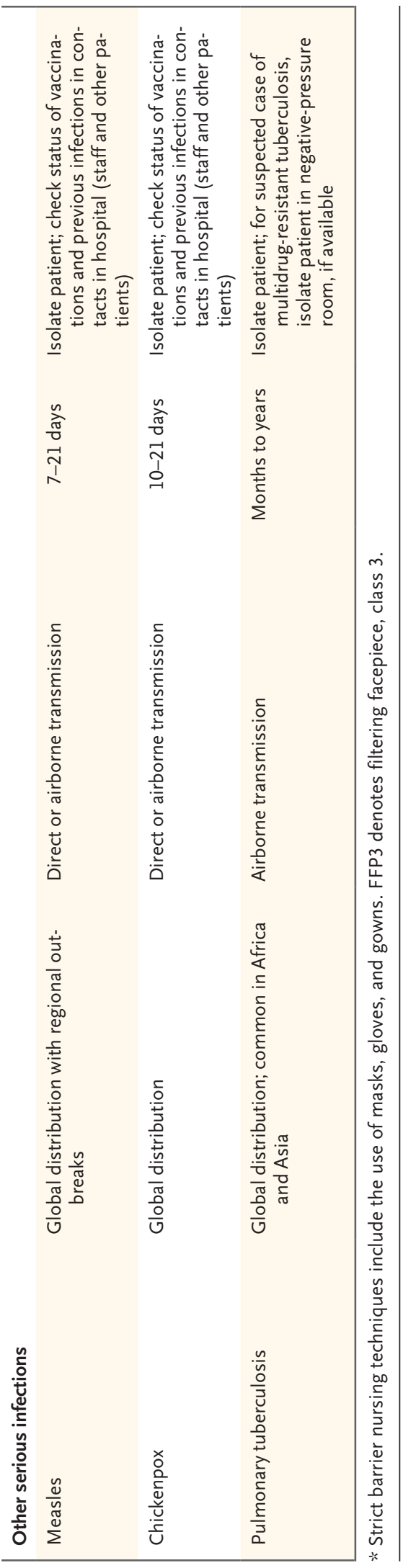

immediately if they might have a life-threatening and potentially transmissible infection. Patients transferred from a health care facility in another country may also need to be isolated before it has been established whether they are colonized or infected with drug-resistant bacteria. Health care workers in direct contact with patients at high risk for a viral hemorrhagic fever or severe airborne infections can reduce their risk of exposure by increasing levels of personal protection, including meticulous hand hygiene, double gloves, fluid-repellent disposable gowns and footwear, a full face shield or goggles, and a fluidrepellent respirator (FFP3 [filtering facepiece, class 3] or N95) for splash and respiratory protection. Negative-pressure isolation reduces the risk of airborne pathogen transmission to other patients and staff. Finally, those responsible for hospital infection control and public health must be informed about all patients with a suspected or confirmed life-threatening infection with the potential for person-to-person transmission.

\section{APPROACH TO LOWER-RISK TRAVELERS WITH FEVER}

Once the life-threatening and transmissible causes of fever in a returning traveler have been ruled out, there is a long list of other possible causes of fever, not all of which are related to travel in tropical regions or to an infectious disease (Fig. 1, and Table S2 in the Supplementary Appendix). ${ }^{45}$ However, the number of possible travel-related infectious causes can be reduced substantially by identifying possible exposures and applying knowledge of the incubation periods for various infections. A detailed travel and activity history is essential. ${ }^{46}$ Different geographic regions are associated with different presentations; systemic febrile illness without localizing findings is common among travelers returning from sub-Saharan Africa or Southeast Asia, acute diarrhea is common among those returning from South Asia and Central Asia, and dermatologic problems are common among those returning from the Caribbean, Central America, or South America. ${ }^{47}$ Travel in temperate regions may also be associated with specific tickborne infections, such as Lyme disease, tularemia, babesiosis, and spotted fevers in parts of the United States and other diseases in eastern Europe and Russia. Each country visited, with dates, must be determined and activity-based risks identified, including types of accommodation; food eaten; exposure to animals, fresh water, and 
bloodborne diseases (e.g., through tattoos or dental work); insect bites; and sexual activity. Contact with ill persons, including those visited in a hospital, may provide important links to disease outbreaks.

The reasons for travel, together with the traveler's age, sex, and immune status, may influence the risks and possible causes of fever. Travelers who visit friends or relatives are at greater risk for malaria, enteric fever, nondiarrheal intestinal parasitic infections, respiratory syndromes, tuberculosis, and sexually transmitted diseases than other travelers. ${ }^{48,49}$ Expatriates are at disproportionately greater risk for chronic infections, including malaria, filariasis, schistosomiasis, strongyloidiasis, giardiasis, brucellosis, and tuberculosis than are short-term travelers. ${ }^{50}$ Travelers older than 60 years of age have proportionally more lifethreatening illnesses, especially severe malaria and lower respiratory tract infections, ${ }^{5}$ than children, in whom diarrheal, skin, and upper respiratory tract infections account for the majority of reported diagnoses. ${ }^{51}$ How sex influences infection susceptibility and reporting patterns is uncertain, but women appear to be proportionally more likely than men to report acute or chronic diarrhea and upper respiratory tract infection and less likely to report vectorborne diseases, such as malaria or rickettsioses..$^{52}$ Opportunistic infections in travelers who are immunocompromised further complicate the clinical assessment. Travelers taking immunosuppressive drugs or glucocorticoids and travelers with poorly controlled human immunodeficiency virus infection are at increased risk for infections (e.g., talaromycosis in Southeast Asia and leishmaniasis in southern Europe, South Asia, and South America).

The majority of febrile illnesses related to exposures during travel develop within a month. ${ }^{3}$ Knowledge of the incubation periods of the suspected infectious diseases can be used to narrow the differential diagnosis. For example, if fever begins more than 21 days after a traveler's return, then dengue, rickettsial infections, Zika virus infection, and viral hemorrhagic fevers are unlikely, regardless of the traveler's exposure history. Infectious causes may be further narrowed by pretravel vaccinations and chemoprophylaxis, although neither approach is $100 \%$ effective. The most common vaccine-preventable causes of fever in travelers are influenza, enteric fever, and viral hepatitis. ${ }^{53}$ In the GeoSentinel database, vaccine-preventable diseases are relatively uncommon (accounting for approximately $3 \%$ of cases) but are associated with high rates of hospitalization $(60 \%)$, a finding that supports the effectiveness of pretravel vaccination in reducing the morbidity and mortality from these diseases. ${ }^{10}$

The recent epidemics of Zika virus infection in Latin America, the Caribbean, and the Pacific Islands have caused considerable alarm, particularly because of increasingly strong associations with the Guillain-Barré syndrome and birth defects, such as microcephaly. ${ }^{54,55}$ The presentation of symptomatic acute Zika virus infection is similar to that of a number of other common causes of fever, including dengue and chikungunya (also transmitted by various species of aedes mosquitoes and often sympatric), rickettsial infections, and leptospirosis. Patients with these infections commonly present with fever, headache, arthralgia, and a maculopapular rash (although the rash is rarely associated with leptospirosis). Zika virus infection can cause conjunctivitis, as can chikungunya, and conjunctival suffusion and subconjunctival hemorrhage are common in patients with leptospirosis.

Initial investigations should include a complete blood count; biochemical studies, including tests of liver and renal function; blood culture; rapid diagnostic tests for malaria and dengue; polymerase-chain-reaction (PCR) testing of a plasma sample (e.g., for rickettsia); serologic tests; a chest film; blood-smear examination; and urine tests, including a dipstick test, microscopic examination, culture, and possibly PCR testing (e.g., for Zika virus or leptospira) (Fig. 1, and Table S2 in the Supplementary Appendix). Clinical suspicion may call for more specific investigations, such as stool-sample examination for ova and parasites, intracranial imaging and lumbar puncture in cases of reduced consciousness (including cases of confirmed cerebral malaria), and abdominal or renal tract ultrasonography or computed tomography in cases of jaundice or acute kidney injury. Point-of-care rapid diagnostic tests for several tropical infections are increasingly available, have been well validated, and are particularly widely used for the diagnosis of malaria and dengue. 
FUTURE DEVELOPMENTS AND CONCLUSIONS

The evidence base to inform the physician in making a rapid diagnosis and instituting appropriate treatment of fever in the returning traveler is growing rapidly, thanks to surveillance networks based in travel clinics (e.g., GeoSentinel) and international surveillance networks, many of which report up-to-date information in digital form (e.g., HealthMap). With the spotlight on antimicrobial drug resistance as a global health threat, funding for antimicrobial-resistance networks, such as the WHO Global Antimicrobial Resistance Surveillance System (GLASS) and the recent Fleming Fund initiative (https://wellcome .ac.uk/press-release/fleming-fund-launched -tackle-global-problem-drug-resistant-infection), is increasing and should lead to higher-quality geographic information on the prevalent antibiotic-resistance patterns in countries visited. The collection, processing, and analysis of data are likely to improve with the increasing connectivity associated with these initiatives. Future integration of clinical data from electronic medical records would further enhance real-time surveillance and potentially provide early signals of changes in disease or patterns of antimicrobial drug resistance and early warnings of developing epidemics.

There will be new challenges as well. The rise in popularity of medical tourism will provide increased opportunities for hospital-associated multidrug-resistant pathogens to spread to hospitals and communities in the traveler's home country, and the continuing rise in travel in general, particularly among the elderly, will increase the cross-border movement of infections. We can expect continued expansion in resources to aid physicians in the treatment of returning travelers, allowing for further improvements in evidencebased diagnosis and management.

No potential conflict of interest relevant to this article was reported.

Disclosure forms provided by the authors are available with the full text of this article at NEJM.org.

\section{REFERENCES}

1. Wilson ME, Freedman DO. Etiology of travel-related fever. Curr Opin Infect Dis 2007;20:449-53.

2. Hill DR. Health problems in a large cohort of Americans traveling to developing countries. J Travel Med 2000;7:259-66. 3. Wilson ME, Weld LH, Boggild A, et al. Fever in returned travelers: results from the GeoSentinel Surveillance Network. Clin Infect Dis 2007;44:1560-8.

4. International tourist arrivals up $4 \%$ reach a record 1.2 billion in 2015. Press release of the United Nations World Tourism Organization, January 18, 2016 (http://media.unwto.org/press-release/ 2016-01-18/international-tourist-arrivals4-reach-record-12-billion-2015).

5. Gautret P, Gaudart J, Leder K, et al. Travel-associated illness in older adults (>60 y). J Travel Med 2012;19:169-77.

6. Molton JS, Tambyah PA, Ang BS, Ling ML, Fisher DA. The global spread of healthcare-associated multidrug-resistant bacteria: a perspective from Asia. Clin Infect Dis 2013;56:1310-8.

7. Nüesch-Inderbinen $M$, Abgottspon $H$, Sägesser G, Cernela N, Stephan R. Antimicrobial susceptibility of travel-related Salmonella enterica serovar Typhi isolates detected in Switzerland (2002-2013) and molecular characterization of quinolone resistant isolates. BMC Infect Dis 2015; 15:212.

8. Marano C, Freedman DO. Global health surveillance and travelers' health. Curr Opin Infect Dis 2009;22:423-9.

9. Leder K. Travelers as a sentinel population: use of sentinel networks to inform pretravel and posttravel evaluation. Curr Infect Dis Rep 2009;11:51-8.

10. Torresi J, Leder K. Defining infections in international travellers through the GeoSentinel surveillance network. Nat Rev Microbiol 2009;7:895-901.

11. Jensenius M, Han PV, Schlagenhauf P, et al. Acute and potentially life-threatening tropical diseases in western travelers - a GeoSentinel multicenter study, 19962011. Am J Trop Med Hyg 2013;88:397-404. 12. Boggild AK, Esposito DH, Kozarsky $\mathrm{PE}$, et al. Differential diagnosis of illness in travelers arriving from Sierra Leone, Liberia, or Guinea: a cross-sectional study from the GeoSentinel Surveillance Network. Ann Intern Med 2015;162:757-64.

13. Schlagenhauf P, Weld L, Goorhuis A, et al. Travel-associated infection presenting in Europe (2008-12): an analysis of EuroTravNet longitudinal, surveillance data, and evaluation of the effect of the pre-travel consultation. Lancet Infect Dis 2015;15:55-64.

14. Brownstein JS, Freifeld CC, Reis BY, Mandl KD. Surveillance Sans Frontières: Internet-based emerging infectious disease intelligence and the HealthMap project. PLoS Med 2008;5(7):e151.

15. Brownstein JS, Freifeld CC, Chan EH, et al. Information technology and global surveillance of cases of 2009 H1N1 influenza. N Engl J Med 2010;362:1731-5.

16. Ambresin AE, D'Acremont V, Mueller Y, Martin O, Burnand B, Genton B. www. fevertravel.ch: an online study prototype to evaluate the safety and feasibility of computerized guidelines for fever in returning travellers and migrants. Comput Methods Programs Biomed 2007;85:1931.

17. Mueller Y, D'Acremont V, Ambresin $\mathrm{AE}$, et al. Feasibility and clinical outcomes when using practice guidelines for evaluation of fever in returning travelers and migrants: a validation study. J Travel Med 2014;21:169-82.

18. Demeester RP, Bottieau E, Pini A, et al. Prospective multicenter evaluation of the expert system "KABISA TRAVEL" in diagnosing febrile illnesses occurring after a stay in the tropics. J Travel Med 2011; 18:386-94.

19. Edberg SC. Global Infectious Diseases and Epidemiology Network (GIDEON): a World Wide Web-based program for diagnosis and informatics in infectious diseases. Clin Infect Dis 2005;40:123-6.

20. Ross JJ, Shapiro DS. Evaluation of the computer program GIDEON (Global Infectious Disease and Epidemiology Network) for the diagnosis of fever in patients admitted to a medical service. Clin Infect Dis 1998;26:766-7. 
21. Luo RF, Bartlett JG. Use of the computer program GIDEON at an inpatient infectious diseases consultation service. Clin Infect Dis 2006;42:157-8.

22. Kimura M, Sakamoto M, Adachi T, Sagara H. Diagnosis of febrile illnesses in returned travelers using the PC software GIDEON. Travel Med Infect Dis 2005;3: $157-60$.

23. Bottieau E, Moreira J, Clerinx J, Colebunders R, Van Gompel A, Van den Ende J. Evaluation of the GIDEON expert computer program for the diagnosis of imported febrile illnesses. Med Decis Making 2008;28:435-42.

24. Gherardin A, Sisson J. Assessing fever in the returned traveller. Aust Prescr 2012;35:10-4.

25. Bottieau E, Clerinx J, Schrooten W, et al. Etiology and outcome of fever after a stay in the tropics. Arch Intern Med 2006; 166:1642-8.

26. O'Brien DP, Leder K, Matchett E, Brown GV, Torresi J. Illness in returned travelers and immigrants/refugees: the 6-year experience of two Australian infectious diseases units. J Travel Med 2006; 13:145-52.

27. Singer $M$, Deutschman CS, Seymour $\mathrm{CW}$, et al. The third international consensus definitions for sepsis and septic shock (Sepsis-3). JAMA 2016;315:801-10.

28. Taylor AJ, Paris DH, Newton PN. A Systematic Review of Mortality from Untreated Scrub Typhus (Orientia tsutsugamushi). PLoS Negl Trop Dis 2015;9(8): e0003971.

29. Kundavaram AP, Jonathan AJ, Nathaniel SD, Varghese GM. Eschar in scrub typhus: a valuable clue to the diagnosis. J Postgrad Med 2013;59:177-8.

30. Dondorp A, Nosten F, Stepniewska K, Day N, White N. Artesunate versus quinine for treatment of severe falciparum malaria: a randomised trial. Lancet 2005; 366:717-25.

31. Dondorp AM, Fanello CI, Hendriksen IC, et al. Artesunate versus quinine in the treatment of severe falciparum malaria in African children (AQUAMAT): an openlabel, randomised trial. Lancet 2010;376: 1647-57.

32. Artesunate is available to treat severe malaria in the United States. Atlanta: Centers for Disease Control and Prevention, 2010 (www.cdc.gov/malaria/diagnosis_treatment/artesunate.html).
33. Kantele A, Lääveri T, Mero S, et al. Antimicrobials increase travelers' risk of colonization by extended-spectrum betalactamase-producing Enterobacteriaceae. Clin Infect Dis 2015;60:837-46.

34. Pitman MC, Luck T, Marshall CS, Anstey NM, Ward L, Currie BJ. Intravenous therapy duration and outcomes in melioidosis: a new treatment paradigm. PLoS Negl Trop Dis 2015;9(3):e0003586.

35. Rahi M, Gupte MD, Bhargava A, Varghese GM, Arora R. DHR-ICMR guidelines for diagnosis \& management of rickettsial diseases in India. Indian J Med Res 2015;141:417-22.

36. Ftika L, Maltezou HC. Viral haemorrhagic fevers in healthcare settings. J Hosp Infect 2013;83:185-92.

37. Ebola (Ebola virus disease): epidemiologic risk factors to consider when evaluating a person for exposure to Ebola virus. Atlanta: Centers for Disease Control and Prevention, May 28, 2015 (https:// www.cdc.gov/vhf/ebola/exposure/risk -factors-when-evaluating-person-for -exposure.html)

38. Public Health England. Risk assessment of the Ebola virus disease outbreak in West Africa: updated 24 June 2015 (https://www.gov.uk/government/ uploads/system/uploads/attachment _data/file/437990/PHE_Risk_Assessment _Update_7_FINAL.pdf).

39. Rogstad KE, Tunbridge A. Ebola virus as a sexually transmitted infection. Curr Opin Infect Dis 2015;28:83-5.

40. Nielsen CF, Kidd S, Sillah AR, Davis E, Mermin J, Kilmarx PH. Improving burial practices and cemetery management during an Ebola virus disease epidemic - Sierra Leone, 2014. MMWR Morb Mortal Wkly Rep 2015;64:20-7.

41. Public Health England. Viral haemorrhagic fever: ACDP algorithm and guidance on management of patients. November 19, 2015 (https://www.gov.uk/government/ publications/viral-haemorrhagic-fever -algorithm-and-guidance-on-management -of-patients)

42. Hui DS, Zumla A. Emerging respiratory tract viral infections. Curr Opin Pulm Med 2015;21:284-92.

43. Ruppé E, Armand-Lefèvre L, Estellat C, et al. High rate of acquisition but short duration of carriage of multidrug-resistant Enterobacteriaceae after travel to the tropics. Clin Infect Dis 2015;61:593-600.
44. van der Bij AK, Pitout JD. The role of international travel in the worldwide spread of multiresistant Enterobacteriaceae. J Antimicrob Chemother 2012;67: 2090-100.

45. Ryan ET, Wilson ME, Kain KC. Illness after international travel. $\mathrm{N}$ Engl J Med 2002;347:505-16

46. Johnston V, Stockley JM, Dockrell D, et al. Fever in returned travellers presenting in the United Kingdom: recommendations for investigation and initial management. J Infect 2009;59:1-18.

47. Freedman DO, Weld LH, Kozarsky PE, et al. Spectrum of disease and relation to place of exposure among ill returned travelers. N Engl J Med 2006;354:119-30.

48. Leder K, Tong S, Weld L, et al. Illness in travelers visiting friends and relatives: a review of the GeoSentinel Surveillance Network. Clin Infect Dis 2006;43:1185-93. 49. Matteelli A, Schlagenhauf $P$, Carvalho AC, et al. Travel-associated sexually transmitted infections: an observational crosssectional study of the GeoSentinel surveillance database. Lancet Infect Dis 2013;13:205-13.

50. Lim PL, Han P, Chen LH, et al. Expatriates ill after travel: results from the Geosentinel Surveillance Network. BMC Infect Dis 2012;12:386.

51. Hagmann S, Neugebauer R, Schwartz $\mathrm{E}$, et al. Illness in children after international travel: analysis from the GeoSentinel Surveillance Network. Pediatrics 2010;125(5):e1072-e1080.

52. Schlagenhauf P, Chen LH, Wilson ME, et al. Sex and gender differences in travelassociated disease. Clin Infect Dis 2010; 50:826-32.

53. Boggild AK, Castelli F, Gautret P, et al. Vaccine preventable diseases in returned international travelers: results from the GeoSentinel Surveillance Network. Vaccine 2010;28:7389-95.

54. Moulin E, Selby K, Cherpillod P, Kaiser L, Boillat-Blanco N. Simultaneous outbreaks of dengue, chikungunya and Zika virus infections: diagnosis challenge in a returning traveller with nonspecific febrile illness. New Microbes New Infect 2016;11:6-7.

55. Wikan N, Smith DR. Zika virus: history of a newly emerging arbovirus. Lancet Infect Dis 2016;16(7):e119-e126. Copyright @) 2017 Massachusetts Medical Society. 